\title{
Technè
}

La science au service de l'histoire de l'art et de la préservation des biens culturels

47 | 2019

Bernard Palissy : nouveaux regards sur la céramique française auX $X \mathrm{VI}^{\mathrm{e}}$ et $\mathrm{XVII}{ }^{\mathrm{e}}$ siècles

\section{Du modèle au tirage : le moulage dans l'œuvre de Bernard Palissy}

From model to mould: casting in the works of Bernard Palissy

\section{Aurélie Gerbier}

\section{(2) OpenEdition}

12 Journals

Édition électronique

URL : http://journals.openedition.org/techne/1448

DOI : $10.4000 /$ techne. 1448

ISSN : 2534-5168

Éditeur

C2RMF

\section{Édition imprimée}

Date de publication : 1 juin 2019

Pagination : 30-32

ISBN : 978-2-11-152830-7

ISSN : $1254-7867$

\section{Référence électronique}

Aurélie Gerbier, " Du modèle au tirage : le moulage dans l'œuvre de Bernard Palissy », Technè [En ligne], 47 | 2019, mis en ligne le 01 juin 2020, consulté le 26 juillet 2020. URL : http://

journals.openedition.org/techne/1448; DOI : https://doi.org/10.4000/techne.1448

\section{(c) (i) $\odot$}

La revue Technè. La science au service de l'histoire de l'art et de la préservation des biens culturels est mise à disposition selon les termes de la Licence Creative Commons Attribution - Pas d'Utilisation Commerciale - Pas de Modification 4.0 International. 
Aurélie Gerbier

\section{Du modèle au tirage : le moulage dans l'œuvre de Bernard Palissy}

From model to mould: casting in the works of Bernard Palissy

\begin{abstract}
Résumé. Le procédé de moulage sur nature, principale technique employée par Bernard Palissy pour réaliser ses plats à rustiques figulines et le décor de ses grottes en céramique glaçurée, est aujourd'hui mieux compris grâce à l'étude de son fonds d'atelier. Mots-clés. Moulage, plâtre, rustiques figulines, grotte.
\end{abstract}

\begin{abstract}
A study made on the contents of Bernard Palissy's workshop allows us to understand better the life cast process, the principal technique he used to make his dishes with rustiques figulines, or rusticware, and to decorate his grottoes in glazed ceramics.
\end{abstract}

Keywords. Casting, plaster, rustiques figulines, grotto.
Bernard Palissy s'exprime volontiers sur les intentions qui animent sa démarche créatrice ou sur les qualités qu'il exige de ses argiles, mais peu sur les techniques qu'il emploie pour réaliser ses rustiques figulines ou les éléments de grotte. Sa quête effrénée de la recette des glaçures relègue au second plan la question du moulage sur nature, technique qui caractérise la grande majorité de sa production. Dans ses écrits, Palissy emploie le terme d'« insculpture » pour qualifier ses réalisations : «il n'y a pas jusques aux petis nerfs, arteres, \& petites costes (...), que l'ouvrier n'ayt observé en son insculpture $^{1}$ ", écrit-il dans l'Architecture et ordonnance de la grotte rustique publiée en 1563. Est-ce une simple confusion de vocabulaire, comme le comprend Isabelle Perrin ${ }^{2}$, ou une opacité souhaitée par l'auteur? Son apprentissage de la technique s'est sans doute fait au contact répété d'autres artisans (voir l'article de P. H. Smith dans ce volume). Un tel processus ne semble pas correspondre à la démarche empirique et solitaire, entre observation et reproduction du phénomène qu'il étudie, qu'il applique à ses autres sujets de recherche que sont les sciences naturelles ou la fabrication des glaçures. Voilà peut-être la clé de son silence. Ce sont donc les conclusions tirées de l'étude de son fonds d'atelier et des pièces de musées qui aident à restituer les différentes étapes de création de ses œuvres.

La technique du moulage sur nature employée par Bernard Palissy lui permet de constituer un répertoire de formes et de décors qu'il agence à volonté. À partir d'animaux, de végétaux, de coquillages ou de minéraux, il réalise un moule en plâtre, dont la fluidité garantit la prise d'empreinte des détails les plus fins. De ce premier moule, il tire un positif ${ }^{3}$ en terre cuite. L'étude des fragments archéologiques a confirmé que Palissy pouvait produire, à partir de ce positif, un second moule en terre ou contre-moule, matériau moins fragile que le plâtre et donc d'utilisation plus longue, ainsi qu'un certain nombre de tirages. Cette séquence a pu être en partie reconstituée pour des motifs animaliers, de grenouille (fig. 1), de lézard ou autre serpent, mais elle demeure encore incomplète pour les éléments végétaux par exemple. Bernard Palissy applique la technique du moulage sur nature aux feuilles, aux céréales, aux fruits et légumes et aux plantes aromatiques. Seules les fleurs, plus délicates et plus fragiles, semblent lui avoir résisté. En témoigne le seul exemple trouvé dans l'atelier du Carrousel, un oeillet ${ }^{4}$. Selon certains observateurs, Palissy aurait également eu recours au modèle humain. Les moules en plâtre de termes anthropomorphes découverts lors des fouilles du XIX ${ }^{\mathrm{e}}$ siècle pourraient en effet avoir été réalisés à partir de cadavres ${ }^{5}$.

Variant l'agencement de ses modèles, Bernard Palissy compose le décor de ses plats rustiques et de ses grottes à partir de ce catalogue de positifs. Pour les pièces de forme, il dispose les éléments sur un support en terre à la forme de l'objet définitif, un bassin par exemple ${ }^{6}$. La composition est à son tour moulée, créant alors une matrice qui pourra servir à plusieurs reprises. Les fouilles du Carrousel ont mis au jour un tel moule (voir fig. 3 de l'article de A. Gerbier dans ce volume) qui a pu être rapproché de deux bassins rustiques, confortant ainsi leur attribution (voir fig. 3 de l'article de P. H. Smith et fig. 3 de l'article de A. Bouquillon et al. dans ce

Aurélie Gerbier, conservateur du patrimoine au musée national de la Renaissance - château d'Écouen (aurelie.gerbier@culture.gouv.fr). 


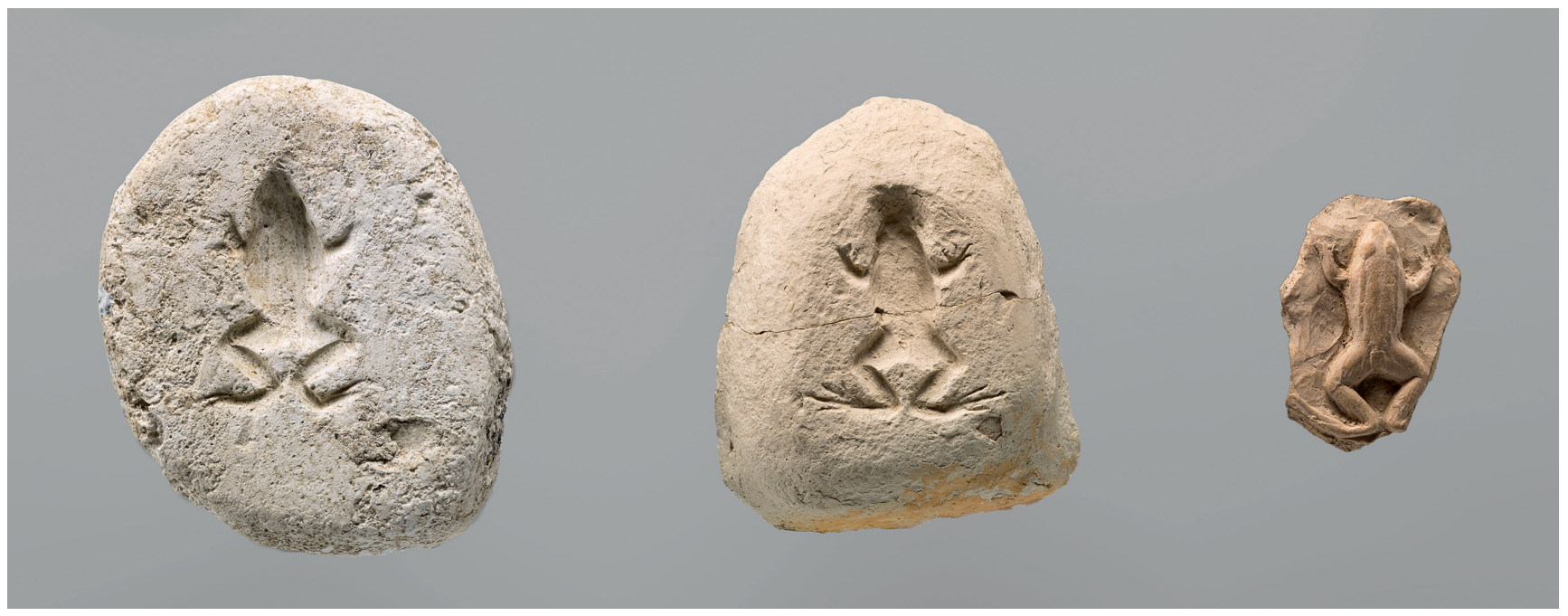

Fig. 1. Atelier de Bernard Palissy, Paris, fouilles des Tuileries, troisième quart du Xvi ${ }^{\mathrm{e}}$ siècle, Deux moules et un tirage de grenouille, plâtre et terre cuite, Écouen, musée national de la Renaissance, EP 2662, EP 1422 et EP 2102. (C) RMN-Grand Palais (musée national de la Renaissance, château d'Écouen)/Mathieu Rabeau.

volume $)^{7}$. Pour les éléments de la grotte, le principe est le même, mais l'exécution légèrement différente notamment pour des raisons d'échelle. Palissy conçoit pour ces projets un décor modulaire, reposant sur la juxtaposition de briques (fig. 2). Sur une structure alvéolaire, il vient apposer une plaque d'argile en positif obtenue selon la technique décrite ci-dessus (fig. 3), le tout étant solidarisé à la barbotine. Entre autres avantages, cette méthode permet à Palissy de sélectionner ses argiles, plus fine pour les parties issues de moulage sur nature afin de garantir la précision des détails, plus dense pour les parties structurelles ${ }^{8}$.

Le geste du sculpteur n'est pas pour autant absent de l'œuvre. Le travail de reprise après moulage apparaît sur certains objets, de même que des traces d'outils peuvent être observées sur certains décors rocheux et aquatiques ${ }^{9}$, notamment sur les briques à décor de rocaille.
Le tirage final est enfin soumis à une première cuisson le préparant à recevoir les glaçures colorées qui, après une seconde cuisson, donneront vie aux rustiques figulines de Palissy.

Si le processus de fabrication des rustiques de Bernard Palissy est aujourd'hui mieux compris, un certain nombre de questions demeurent posées. Lors de la première phase de moulage en plâtre, quel agent démoulant emploie Palissy? Ce dernier a-t-il systématiquement reproduit cette séquence ou, comme le suppose Léonard Amico ${ }^{10}$, l'expérience venant, a-t-il renoncé à la phase de moulage au plâtre ? Le manuscrit Ms. Fr. 640 de la Bibliothèque nationale de France permet d'esquisser de premières réponses (voir l'article de $\mathrm{P}$. H. Smith dans ce volume), qui ne pourront être confirmées que lors d'une étude approfondie des moules en plâtre du fonds d'atelier. 


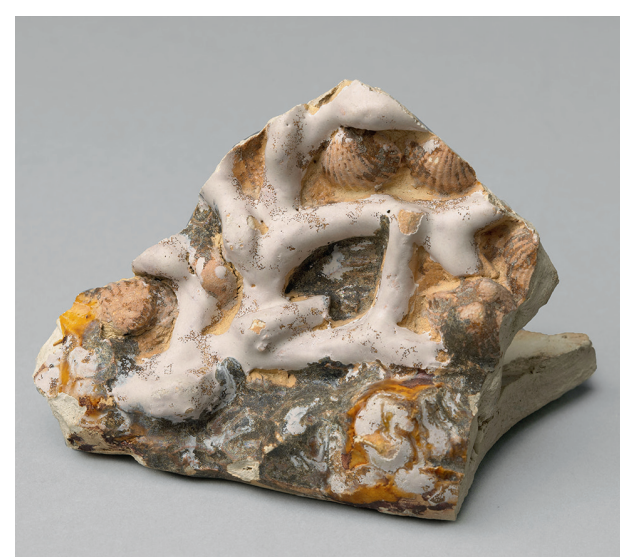

Fig. 2. Atelier de Bernard Palissy, Paris, fouilles des Tuileries, troisième quart du $\mathrm{xvI}^{\mathrm{e}}$ siècle, Brique à décor de corail et coquillages, terre cuite glaçurée, Écouen, musée national de la Renaissance, EP 653. (c) RMN-Grand Palais (musée national de la Renaissance, château d'Écouen)/ René-Gabriel Ojéda.

Fig. 3. Atelier de Bernard Palissy, Paris, fouilles des Tuileries, troisième quart du Xvi ${ }^{\mathrm{e}}$ siècle, Moule de décor rustique, terre cuite, Écouen, musée national de la Renaissance, EP 1402. (C) RMN-Grand Palais (musée national de la Renaissance, château d'Écouen)/ Stéphane Maréchalle.

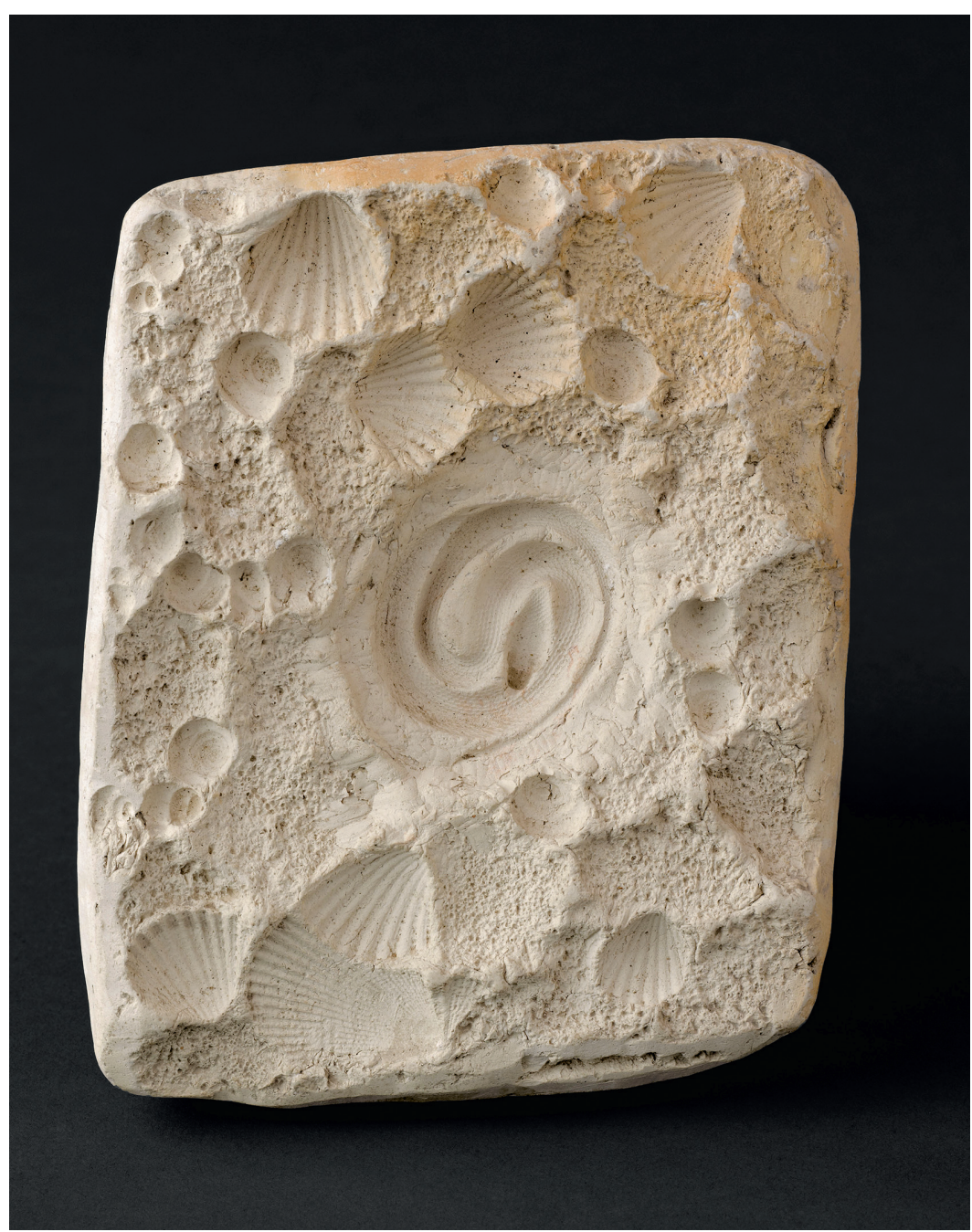

Notes

1. Palissy, 2010, p. 68.

2. Perrin, 2001, p. 129

3. Par convention, on appelle " positif" le tirage, unique, issu du premier moule en plâtre.

4. Écouen, musée national de la Renaissance, EP 546.

5. Berty, Legrand, 1868, p. 47.

6. Le fonds d'atelier de Bernard Palissy contient plusieurs moules, complets pour certains, de plats sans décor, destinés à cet effet.

7. Poulain, 1993.

8. Bouquillon, Barbe, Lehuédé et al. 2013, p. 153-156.

\section{Amico, 1996, p. 94}

10. Amico, 1996, p. 88

Bibliographie sélective

Amico L., 1996, À la recherche du paradis terrestre. Bernard Palissy et ses continuateurs, Flammarion, Paris.

Berty A., Legrand H., 1868, Topographie Historique du Vieux Paris, Région du Louvre et des Tuileries, Paris, tome II.

Bouquillon A., Barbe F., Lehuédé P., Castaing J., Crépin-Leblond T., 2013, "Bernard Palissy: scientist and potter of the Renaissance in France", dans Saunders D., Spring M., Meek A. (eds.), The Renaissance Workshop: The Materials and Techniques of Renaissance Art,

Archetype Publications in association with the British Museum, London, p. 152-159.

Palissy B., 2010, Euvres complètes, Fragonard M.-M. (dir.), seconde édition revue et annotée, Honoré Champion, Paris.

Perrin I., 2001, Les techniques céramiques de Bernard Palissy, thèse sous la direction de M. Jean Guillaume, soutenue en 1998, université Paris IV Sorbonne, Anrt, Lille, 2 vol.

Poulain D., 1993, « Les "rustiques figulines" du musée des Beaux-Arts de Lyon », Bulletin des Musées et Monuments lyonnais, 3-4, p. 24-27. 